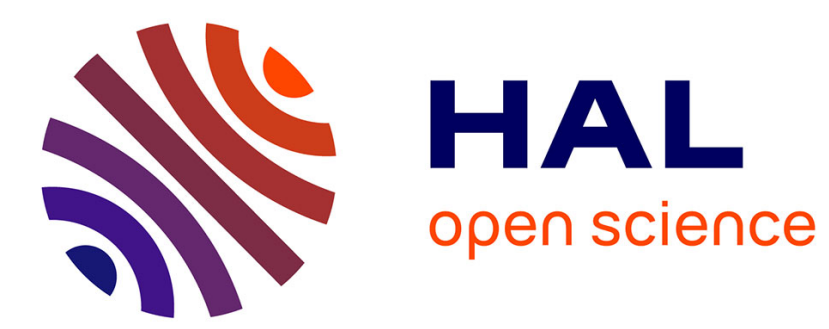

\title{
Imagerie thermique par radiométrie microonde
}

\author{
B. Bocquet, A. Mamouni, J.C. van de Velde, Y. Leroy
}

\section{To cite this version:}

B. Bocquet, A. Mamouni, J.C. van de Velde, Y. Leroy. Imagerie thermique par radiométrie microonde. Revue de Physique Appliquée, 1988, 23 (7), pp.1273-1279. 10.1051/rphysap:019880023070127300 . jpa-00245941

\section{HAL Id: jpa-00245941 https://hal.science/jpa-00245941}

Submitted on 1 Jan 1988

HAL is a multi-disciplinary open access archive for the deposit and dissemination of scientific research documents, whether they are published or not. The documents may come from teaching and research institutions in France or abroad, or from public or private research centers.
L'archive ouverte pluridisciplinaire HAL, est destinée au dépôt et à la diffusion de documents scientifiques de niveau recherche, publiés ou non, émanant des établissements d'enseignement et de recherche français ou étrangers, des laboratoires publics ou privés. 


\title{
Imagerie thermique par radiométrie microonde
}

\author{
B. Bocquet, A. Mamouni, J. C. Van de Velde et Y. Leroy \\ Centre Hyperfréquences et Semiconducteurs, U.A. C.N.R.S. n $^{\circ} 287$, Bât. P4, Université des Sciences et \\ Techniques de Lille Flandres Artois, 59655 Villeneuve d'Ascq Cedex, France
}

(Reçu le 5 novembre 1987, révisé le 14 décembre 1987, accepté le 18 décembre 1987)

\begin{abstract}
Résumé. - La radiométrie ou thermographie microonde (T.M.O.) permet de réaliser des investigations thermologiques non invasives sur les tissus vivants. Elle intéresse des applications liées au diagnostic médical et au contrôle de température en hyperthermie. Nous faisons un bilan des recherches actuelles dans ce domaine. L'état d'avancement de nos travaux nous a permis de mettre au point une méthode d'imagerie thermique microonde (I.T.M.O.) haute résolution. L'interprétation des signaux radiométriques a pour but de réaliser une thermométrie quantitative en volume : nous montrons comment notre système d'imagerie permet de traiter le cas de structures thermogènes de forme compacte.
\end{abstract}

\begin{abstract}
Microwave radiometry or thermography (T.M.O.) enables non invasive thermographic investigations in the living tissues. It concerns applications about medical diagnosis and thermometric control in hyperthermia. We give a review of the actual research in this field. The progress of our works leads to a hight definition microwave thermometric imaging (I.T.M.O.). The interpretation of the radiometric signals gives a quantitative thermometry in volume in the case of compact thermal structures.
\end{abstract}

\section{Introduction.}

La radiométrie microonde, basée sur la mesure du rayonnement thermique naturel émis par les tissus vivants, est le point de départ d'une méthode thermométrique.

L'intérêt principal de cette méthode est dû au caractère atraumatique de la mesure et au fait qu'elle permet de repérer l'existence de gradients thermiques (pour des profondeurs jusque $4 \mathrm{~cm}$ ). Des investigations thermologiques sont effectuées pour mettre au point de nouveaux procédés technologiques intéressant la médecine, en particulier, du point de vue diagnostic et pour un contrôle thermométrique non invasif en hyperthermie.

Après une présentation des bases physiques et technologiques de cette méthode, nous donnons un aperçu des recherches en ce domaine. Nous présentons alors les résultats obtenus par notre groupe de recherche. Ces résultats sont basés sur deux centres d'intérêts :

- un radiomètre multisonde a été construit, permettant d'établir ainsi un processus d'imagerie thermique haute résolution (compte tenu de la longueur d'onde utilisée) ;

- une méthode canonique d'inversion est basée sur le traitement de l'image et sur des mesures radiométriques effectuées à deux fréquences $(1,5$ et $3 \mathrm{GHz}$ ). Elle s'applique au cas de structures thermogènes compactes.

\section{Principe et mise en cuvre de la radiométrie microonde.}

La radiométrie microonde est basée sur le principe du rayonnement du corps noir gouverné par la loi de Planck. La brillance spectrale d'un matériau donné peut s'écrire :

$$
B(T, f)=e(f) \frac{2 h f^{3}}{c^{2}} \frac{1}{\exp (h f / k T)-1}
$$

où $e(f)$ représente l'émissivité du milieu, $T$ sa température absolue et $f$ la fréquence considérée. Les constantes $h, k$ et $c$ sont respectivement les 
constantes de Planck et de Boltzmann, et la vitesse de la lumière.

Aux fréquences de travail que nous utilisons, cette expression se réduit à l'approximation de RayleighJeans $(h f \ll k T)$

$$
B(T, f)=e(f) \frac{2 k T f^{2}}{c^{2}}
$$

montrant une dépendance directe entre puissance de bruit thermique et température du milieu [1].

Une sonde ou antenne, placée au contact du milieu absorbant (les tissus vivants dans le cas d'applications médicales) capte la puissance de bruit émise par les volumes élémentaires situés sous la sonde.

Ces sondes sont ici des ouvertures de guide rectangulaire fonctionnant en mode T.E.O.1 remplis d'un matériau de forte permittivité $\left(\varepsilon_{R}=25\right)$. Dans ces conditions, on minimise le coefficient de réflexion à l'interface avec les tissus tandis que la taille de l'ouverture est réduite, ce qui permet d'augmenter la résolution spatiale associée à la mesure radiométrique.

La puissance transmise par l'un de ces volumes élémentaires à la sonde est proportionnelle :

- au paramètre de couplage sonde-matériau ;

- à la température absolue du volume élémentaire.

Le paramètre de couplage est défini à partir du théorème de réciprocité appliqué au couple d'antennes constituées par la sonde et le volume élémentaire. Ce paramètre est proportionnel à la conductivité du volume élémentaire multipliée par le carré du champ électrique reçu au même point lorsque la sonde est connectée à un générateur microonde de même fréquence. Par conséquent, pour calculer l'intensité d'un signal radiométrique, il est nécessaire de connaître le champ émis par la sonde en tout point du matériau. Il faut ici considérer le rayonnement en champ proche, généralement très différent du mode T.E.M. Ce rayonnement doit être décrit en terme de propagation de plusieurs modes $[2,3]$.

Pour le cas d'un matériau homogène, ce paramètre de couplage décroît avec la distance à l'ouverture de la sonde et pour une distance donnée, il décroît avec la fréquence. Il est à noter que pour les tissus vivants, les paramètres de couplage sont encore significatifs pour des profondeurs de quelques centimètres aux fréquences comprises entre 1 et $6 \mathrm{GHz}$.

Le radiomètre, connecté à la sonde, permet de mesurer la puissance de bruit captée. Il comporte en particulier un amplificateur de grand gain $(\sim 50 \mathrm{~dB})$, de faible facteur de bruit $(<3 \mathrm{~dB})$ et de grande bande passante $(\sim 1 \mathrm{GHz})$ étant donné le faible signal de bruit qui doit être mesuré [4].

Dans ces conditions, le signal radiométrique mesuré pour une position donnée de la sonde peut être associé à une température radiométrique déterminée par un étalonnage préalable, la sonde étant placée sur un milieu à température connue. Le radiomètre est conçu de façon telle que le signal reçu ne varie pas, même s'il se produit une faible modification du coefficient de réflexion à l'interface, [5] ce qui est le cas en pratique.

\section{Bilan des recherches en radiométrie microonde.}

Les pionniers en ce domaine sont Barrett et Myers [6] qui ont construit des radiomètres à 1,3 et $3,3 \mathrm{GHz}$ et ont expérimenté un nouveau procédé de détection de cancer du sein. Cette méthode est basée sur la combinaison des thermographies microonde et infrarouge.

Les premiers systèmes radiométriques construits au laboratoire $[5,7]$ ont conduit à plusieurs séries d'investigations thermologiques $[8,9]$. Une méthode de modélisation a été proposée vers la même époque [2]. Nous avons aussi montré qu'il était possible de combiner le chauffage microonde et la radiométrie dans un même système [10] permettant un meilleur contrôle atraumatique de la température pendant des séances d'hyperthermie. Suite à des expériences sur modèles et à des évaluations cliniques, notre étude a conduit au développement des systèmes Hylcar (hyperthermie avec contrôle atraumatique par radiométrie) par la Société Odam.

A noter également une étude sur la radiométrie par corrélation [11] dont le principe est basé sur la réalisation de la fonction de corrélation des signaux de bruits captés par deux sondes.

D'autres auteurs participent également à des investigations thermologiques. Edrich [12] développa quelques systèmes radiométriques travaillant à 34 et $10 \mathrm{GHz}$ pour lesquels l'émission thermique des tissus est focalisée par un réflecteur elliptique sur un cornet connecté au radiomètre. Il s'agit des seuls cas de radiomètre sans contact pour applications médicales [13].

Carr [14] a réalisé un radiomètre à $4,7 \mathrm{GHz}$ associé à un émetteur de fréquence $915 \mathrm{MHz}$ permettant de chauffer les tissus situés en face de la sonde.

Land [15] a construit plusieurs radiomètres utilisés pour l'étude de pathologies thermogènes telles que cancer du sein, rhumatisme du genou, appendicite.

Paglione [16] a réalisé un radiomètre travaillant à $4 \mathrm{GHz}$ pour la détection de l'appendicite.

Mizushina [17] a construit un radiomètre multifréquences (bandes centrées à $1,5,2,5$ et $3,5 \mathrm{GHz}$ ) utilisé pour des expériences sur modèles et sur animaux dans le but de restituer le profil de température en profondeur.

La littérature cite encore d'autres systèmes radiométriques [18] montrant les espoirs misés sur cette technique. 
4. Un radiomètre multisonde pour une imagerie radiométrique.

Une fois les techniques radiométriques au point, il convient de définir leur exploitation en vue de conduire des investigations en médecine.

La synthèse d'images radiométriques, réalisée à partir des mesures effectuées en de nombreux points d'une surface nous a paru intéressante car elle donne une information qualitative sur la distribution de température présente dans la couche de tissus accessible à la mesure radiométrique. En effet, l'examen de telles images rend possible la localisation de structures thermogènes relatives à certaines pathologies.

Le besoin de réunir un grand nombre de mesures pour l'obtention de ces images nous a conduit à la réalisation d'un radiomètre multisonde [19]. La tête de réception est ainsi constituée de six sondes juxtaposées (Fig. 1a) reliées séquentiellement au radiomètre par un commutateur multivoie. Un microordinateur pilote cette commutation, fait l'acquisition et la sauvegarde des données et réalise une image synthétique. Au-delà de son intérêt en tant que moyen d'examen qualitatif, cette image est à la base d'une reconstruction de profil de température qui sera présentée dans la dernière partie de cet article.

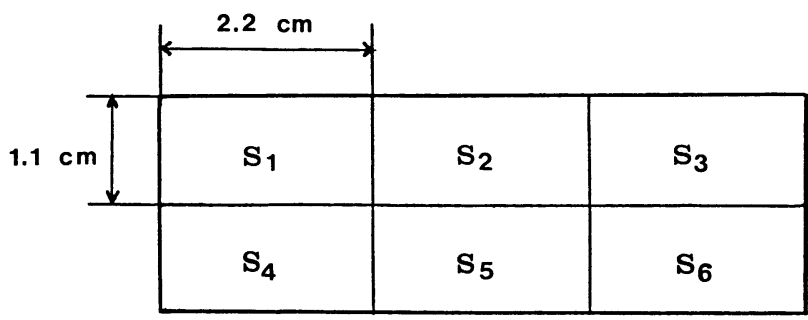

Fig. 1a. - Géométrie des ouvertures de la multisonde.

[Geometry and size of the multiprobe.]

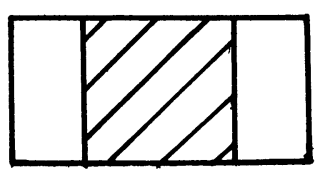

Fig. 1b. - Mise en évidence de la surface la plus sensible (hachurée) d'une ouverture de sonde.

[Footprint of the probe aperture (dashed area).]

La synthèse d'une image radiométrique nécessite au préalable la connaissance de l'étendue du volume participant au signal recueilli pour une position donnée de la sonde. Cette détermination est possible, par application du théorème de réciprocité, suite à un calcul préalable de la carte des champs rayonnés. Elle est faite à partir d'une méthode modale basée sur la résolution d'équations intégrales à la discontinuité sonde-matériau [3].

A l'issue de ce type d'étude, il s'avère que l'ouverture rectangulaire des sondes présente une zone plus sensible au centre au détriment de ses parties latérales. Le positionnement des sondes, lors des mesures radiométriques, doit prendre en compte cette caractéristique. C'est la raison pour laquelle nous avons imaginé un processus mettant en œuvre un recouvrement partiel des surfaces couvertes par les sondes, au cours de leurs déplacements successifs.

Sur la figure 2, nous figurons la méthode de déplacement permettant d'obtenir 72 données radiométriques pour une surface d'investigation de $6,6 \times 6,6 \mathrm{~cm}$ : ce résultat s'acquiert au moyen de 12 positionnements de la multisonde.

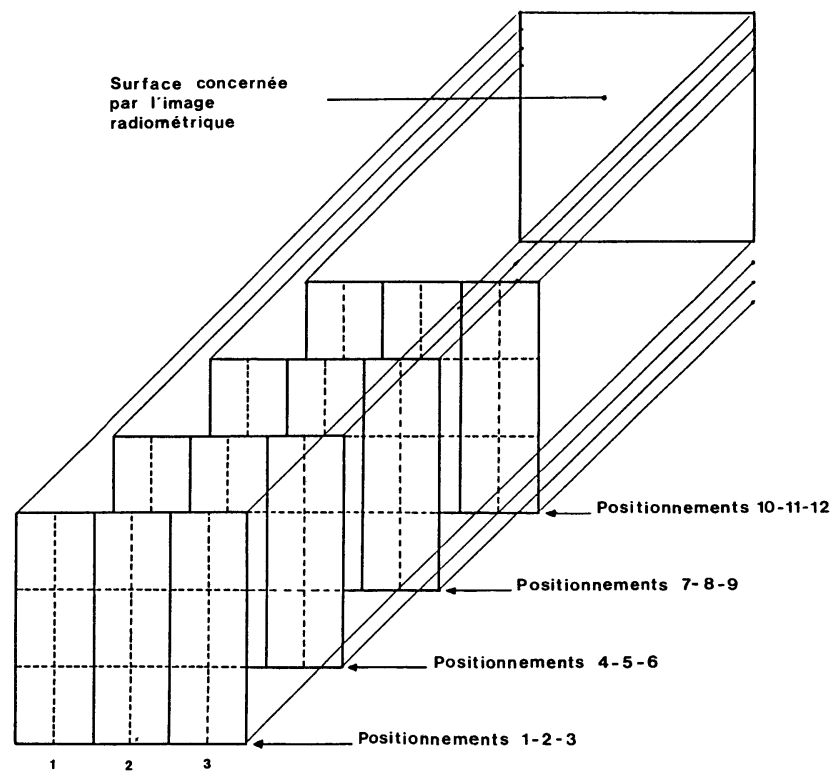

Fig. 2. - Séquences de positionnement de la multisonde pour la réalisation d'une image radiométrique de 72 points.

[Mode of positionnings of the multiprobe for a 72 points radiometric imaging.]

Notre méthode de calcul des signaux radiométriques nous renseigne sur la géométrie du volume participant au signal radiométrique. Celui-ci dépend des propriétés diélectriques des tissus. Dans le cas de tissus à forte teneur en eau à $3 \mathrm{GHz}$ nous savons que $70 \%$ de la contribution totale des paramètres de couplage sont relatifs au matériau situé face à la zone hachurée de l'ouverture (Fig. 1b). Nous prenons actuellement ce résultat comme base de travail. L'obtention de l'image radiométrique se fait ainsi par interpolation des données en tenant compte de cette pondération.

La figure 2 représente un recouvrement en mode vertical où les grands côtés de la sonde sont verti- 
caux. On peut aussi définir un mode horizontal en faisant pivoter les sondes de $90^{\circ}$, les grands côtés étant alors horizontaux. Nous avons montré sur des modèles que la combinaison des deux modes précités permettait une résolution spatiale intéressante. En effet, 144 points de mesure réalisés sur la surface d'investigation correspondent sensiblement à une mesure tous les $5 \mathrm{~mm}$ ce qui donne un ordre de grandeur de la résolution spatiale de l'image. Dans le cadre des évaluations cliniques, nous avons pu constater que si l'ensemble des mesures radiométriques correspondant à une image est compris dans un intervalle inférieur à $1,5^{\circ}$, il est nécessaire de disposer de 144 données radiométriqques pour réaliser une image significative.

Les évaluations cliniques, effectuées avec ce procédé, sont basées sur la comparaison de deux images radiométriques. La première provient d'une zone de tissus, en général pathologique, par exemple, dans le but de mettre en évidence des points chauds. La seconde image, correspondant à une surface de même étendue choisie sur des tissus sains et, en général, symétrique de la zone précédente, est prise

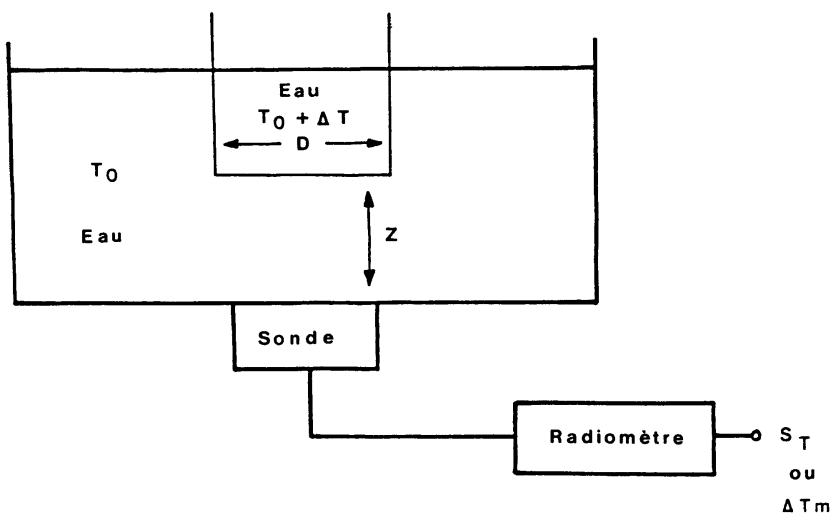

Fig. 3. - Modèle expérimental utilisé pour les mesures radiométriques de structures compactes.

[Experimental set up used for radiometric measurements of a compact thermal structures.]

comme référence. La gamme située entre les températures extrêmes correspondant aux deux images sert à définir les 16 pseudocouleurs donnant une échelle dynamique des mesures radiométriques.

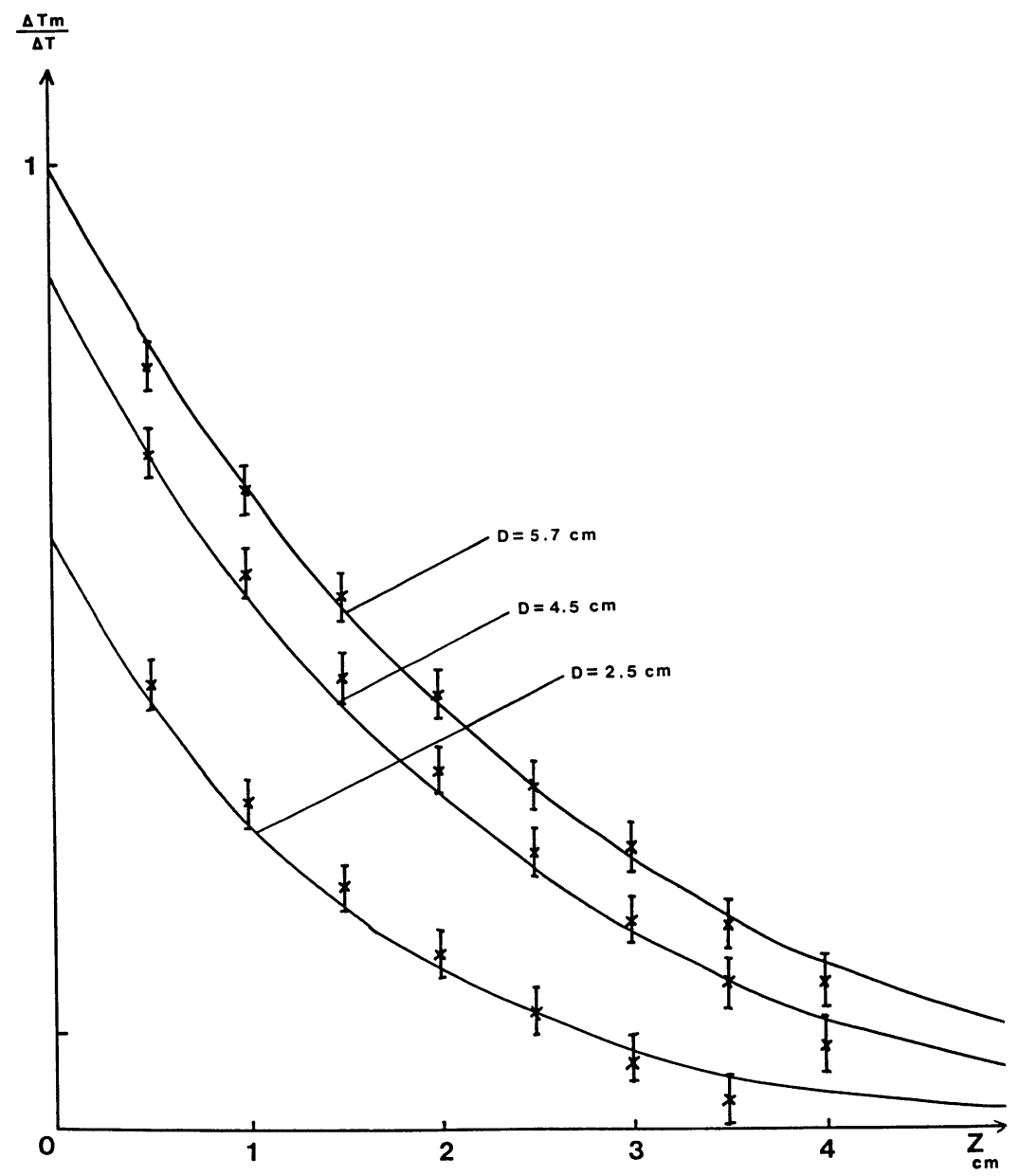

Fig. 4. - Signaux radiométriques obtenus sur l'eau à $f=1,5 \mathrm{GHz}\left(T_{0}=33^{\circ} \mathrm{C}, \Delta T=5^{\circ} \mathrm{C}\right)$.

[Radiometric signals obtained on water at $f=1.5 \mathrm{GHz}\left(T_{0}=33^{\circ} \mathrm{C}, \Delta T=5^{\circ} \mathrm{C}\right)$. 
Ce processus d'imagerie est actuellement utilisé au Centre Anti-cancéreux Oscar-Lambret à Lille pour la détection des cancers du sein (Pr. Giaux, Dr. Delannoy, Dr. Delvallée).

Une autre campagne d'expérimentation a été faite au C.E.A. - Laboratoire de Radiobiologie - à Jouy en Josas sur des porcs afin d'observer l'évolution d'érythèmes causés par une irradiation locale aiguë provenant de sources nucléaires [20, 21]. Les conclusions de ces études montrent que la thermographie microonde est un procédé efficace qui permet de suivre l'évolution thermique de la lésion et de tester des médicaments.

\section{Interprétation des données radiométriques.}

La signification physique des signaux radiométriques posée en terme de reconstitution de profils thermiques est un problème qui est posé depuis les débuts de la radiométrie et qui n'est pas encore complètement résolu. En effet, la solution d'un tel problème pour des profils de température complexes passe par le traitement d'un grand nombre de mesures radiométriques effectuées en différents points de la surface sous investigation et à plusieurs fréquences.

Edenhoffer [22] et Schaller [23] ont traité le cas d'une inversion de données radiométriques à 3 fréquences sur un matériau multicouche (peau, graisse, muscle) par une méthode d'optimisation.

Chive et al. [24] se sont intéressés à la reconstitution du profil de température sur l'axe d'une sonde durant une séance d'hyperthermie à partir de la connaissance de la température de surface, de mesures radiométriques à deux fréquences et par application de l'équation des transferts de chaleur.

Mizushina et al. [17] obtiennent la température en fonction de la profondeur sur des modèles et sur des animaux à l'aide de mesures radiométriques effectuées à trois fréquences. Ils supposent, a priori, que l'évolution de la température varie exponentiellement avec la profondeur. Pour les profondeurs inférieures à $5 \mathrm{~cm}$, l'erreur est inférieure à $\pm 0,5^{\circ} \mathrm{C}$.

Bardatti et al. $[25,26]$ traitent le problème de l'inversion des signaux. Actuellement, ils reconstituent un objet thermique bidimensionnel de forme

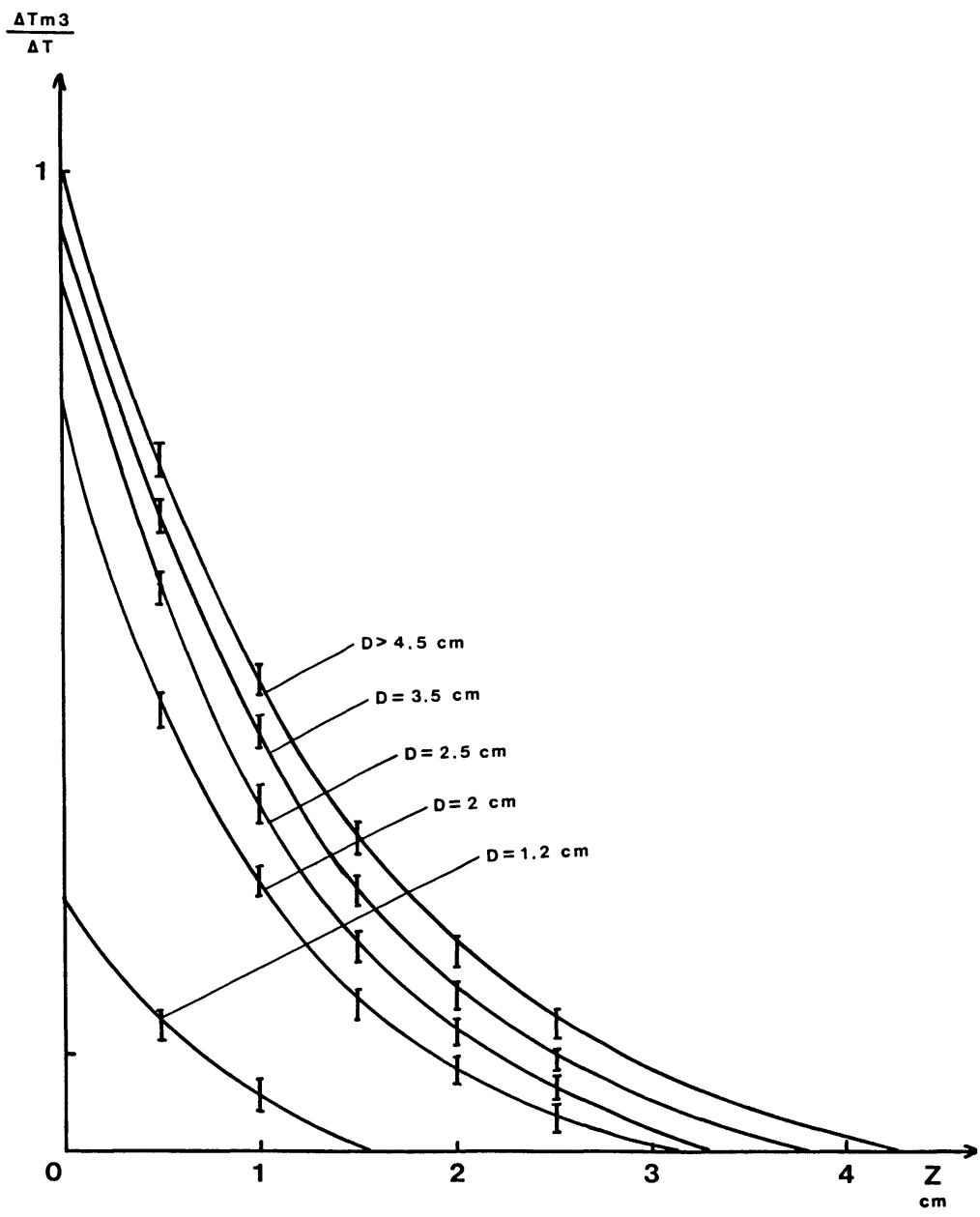

Fig. 5. - Signaux radiométriques obtenus sur l'eau à $f=3 \mathrm{GHz}\left(T_{0}=33^{\circ} \mathrm{C}, \Delta T=5^{\circ} \mathrm{C}\right)$.

[Radiometric signals obtained on water at $f=3 \mathrm{GHz}\left(T_{0}=33^{\circ} \mathrm{C}, \Delta T=5^{\circ} \mathrm{C}\right)$.] 
ramassée à partir du traitement des mesures réalisées à quatre fréquences.

Nous nous sommes aussi intéressé à ce problème en considérant le cas de structures thermiques compactes (cas le plus souvent rencontré). Notre méthode part d'expériences faites sur des modèles.

La première étape de la méthode est basée sur la connaissance de l'image radiométrique à $3 \mathrm{GHz}$ et sur la mesure d'un radiomètre $1,5 \mathrm{GHz}$. Les mesures radiométriques sont réalisées sur des cylindres (diamètres $D$ ) emplis d'eau (température $T_{0}+\Delta T$ ) plongés à une profondeur $z$ dans une cuve contenant le même liquide (température $T_{0}$ ) (Fig. 3) (en général $\Delta T=5^{\circ} \mathrm{C}$ ). La forme de ces objets a été choisie pour des raisons de commodité expérimentale. L'étude de structures plus complexes sera abordée ultérieurement par le calcul.

Le signal radiométrique est défini en terme d'excès de température $\Delta T_{\mathrm{m}}$. Nous avons représenté le rapport $\Delta T_{\mathrm{m}} / \Delta T$ en fonction de la profondeur et du diamètre $D$ à $1,5 \mathrm{GHz}$ (Fig. 4) et $3 \mathrm{GHz}$ (Fig. 5).

Les premières expériences ont conduit à définir les limites de visibilité (rapport signal sur bruit de $0 \mathrm{~dB}$ ), compte tenu de la sensibilité du radiomètre (estimé à $0,1^{\circ} \mathrm{C}$ ) en fonction de $D, z$ et $\Delta T$ [27]. Nous avons montré que les objets sont encore visibles si la profondeur est inférieure à 4,5 et $3 \mathrm{~cm}$ respectivement à 1,5 et $3 \mathrm{GHz}$ et si le diamètre est supérieur à 2 et $1 \mathrm{~cm}$ respectivement à 1,5 et $3 \mathrm{GHz}$.

Nous avons aussi montré que les valeurs de $\Delta T_{\mathrm{m}} / \Delta T$ fonction de $z$ et $D$ peuvent être utilisées pour définir une méthode canonique d'inversion. Cette méthode est d'abord basée sur le fait que l'extraction de contour sur l'image radiométrique à $3 \mathrm{GHz}$ donne directement le diamètre de l'objet. Les courbes $\Delta T_{\mathrm{m}} / \Delta T(D, z)$ à 1,5 et $3 \mathrm{GHz}$ donnent ensuite directement $z$ et $\Delta T$.

Cette méthode a été traduite sous forme d'une modélisation numérique. Les données expérimentales (le diamètre $D$ est déduit de l'extraction de contour de l'image à $3 \mathrm{GHz}$ ) sont introduites dans un processus itératif basé sur la méthode de Newton, qui permet de retrouver les valeurs de $\Delta T$ et $z$, compte tenu de la connaissance des expressions analytiques de $\Delta T_{\mathrm{m}} / \Delta T(D, z)$ à 1,5 et $3 \mathrm{GHz}$.

Un organigramme (Fig. 6) présente les différentes étapes permettant d'arriver au résultat. Les marges d'erreurs sur $D$ sont actuellement d'environ $2 \mathrm{~mm}$; les erreurs sur $\Delta T$ et $z$ dépendent largement de ces paramètres.

\section{Conclusion.}

Dans cet article, nous avons présenté l'état de l'art en ce qui concerne la radiométrie microonde appli-

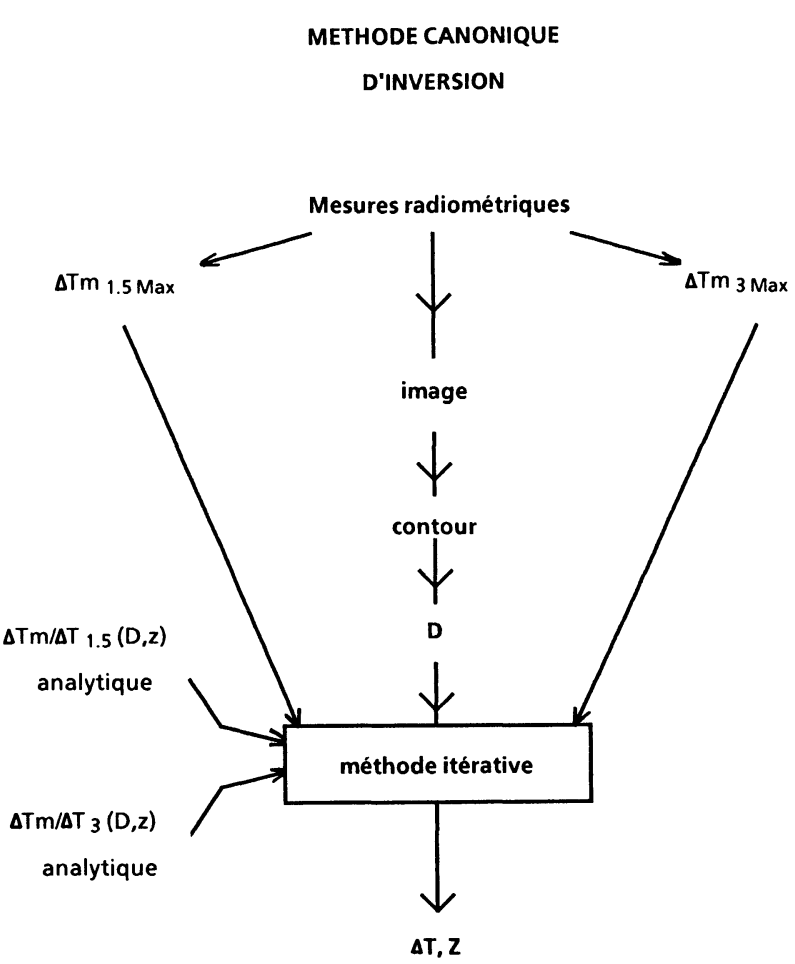

Fig. 6. - Synoptique du programme donnant les paramètres $Z, D$ et $\Delta T$ décrivant une structure thermogène compacte.

[Organigram of the inversion method used to determine the parameters $z, D$ and $\Delta T$ of a compact thermal structure.]

quée en médecine. Ce procédé qui permet des investigations thermométriques non invasives en profondeur intéresse un nombre croissant de laboratoires en ce qui concerne la construction et l'essai de nouveaux systèmes d'évaluations cliniques ainsi que la recherche de méthodes d'inversion des données radiométriques. Nous avons présenté un nouveau procédé de synthèse d'images radiométriques obtenues en différents points (72 ou 144) d'une surface $(6,6 \mathrm{~cm} \times 6,6 \mathrm{~cm})$. Les conditions de visibilité d'une structure thermogène de forme ramassée sont étudiées; sa taille peut être déterminée avec une précision de quelques millimètres. Une méthode canonique d'inversion est également proposée, basée sur des mesures radiométriques à deux fréquences, qui s'applique à de telles structures thermogènes.

\section{Remerciements.}

Ces travaux bénéficient d'aides attribuées par l'Anvar, le M.R.T. et l'Etablissement Public Régional Nord Pas-de-Calais. 


\section{Bibliographie}

[1] Myers, P. C., SAdowsky, N. L., Barret, A. H., Microwave thermography: Principle, Method and Clinical Applications, J. Microwave Power 14 (1979) 105-115.

[2] Robillard, M., Chive, M., Leroy, Y., Audet, J., Pichot, C., Bolomey, J. C., Microwave thermography characteristics of waveguide applications and signatures of thermal structures, $J$. Microwave Power 17 (1982).

[3] Mamouni, A., Van de Velde, J. C., (1987) Résultats à paraître.

[4] Evans, G., MCLeish, C. W., R.F. Radiometer Handbook, Artech House (1977).

[5] Mamouni, A., Bliot, F., Leroy, Y., Moschetto, Y., A modified radiometer for temperature and microwave properties measurements of biological substances, 7th E.M.C. Microw. Exhib. and Publ. Ltd (1977) 703-707.

[6] Barrett, A. H., Myers, P. C., SAdowski, N. L., Detection of breast cancer by microwave radiometry, Radio Science' 12 (1977) 167-171.

[7] LEROY, Y., Microwave radiometry and thermography. Present and prospective, Biomedical Thermology (A.R. Lyss Inc, New York) 1982, p. 485499.

[8] Gautherie, M., Mamouni, A., Samsel, M., GuerQUIN-KERN, J. L., LEROY, Y., GROS, C., Microwave radiothermometry $(9 \mathrm{GHz})$ applied to breast cancer, J. Opt. Photonics appl. Med. 2 (1980) 154-160.

[9] Robert, J., Edrich, J., Mamouni, A., Escanye, J. M., ITTY, C., Microwave thermometry in intracranial pathology, Biomedical thermology (A.R. Lyss Inc, New York) 1982, p. 501-508.

[10] Nguyen, D. D., Mamouni, A., Leroy, Y., ConsTANT, E., Simultaneous microwave local heating and microwave thermography. Possible clinical applications, J. Microwave Power 14 (1979) 135137.

[11] Mamouni, A., Van de Velde, J. C., Leroy, Y., New correlation radiometer for microwave thermography, Electron. Lett. 17 (1981) 554-555.

[12] Edrich, J., Jobe, W. E., Hendee, W. R., CACAK, H. K., GaUtherie, M., Imaging thermograms at $\mathrm{cm}$ and $\mathrm{mm}$ wavelength, Ann. N.Y. Acad. Sci. 335 (1980) 443-455.

[13] EDRICH, J., Microwaves in breast cancer detection, A paraître dans Eur. J. radiol. (1987).

[14] Carr, K., Morsi el-Madhi, A., Schaeffer, J., Dual-mode microwave system to enhance early detection of cancer, IEEE Trans. on M.T.T. 29 (1981) 256-260.
[15] LAND, D. V., Radiometer receivers for microwave thermography, Microwave J. 26 (1983) 196-201.

[16] Paglione, R., Portable diagnostic radiometer, R.C.A. Rev. 47 (1986) 635-643.

[17] Mizushina, S., Yamamura, Y., Siguera, T., Three band microwave radiometer system for non-invasive measurement of the temperature at various depth, Proc. IEEE MTT's Digest (1986) p. 759-762.

[18] IsKander, M. F., DuRney, C. H., Microwave methods of measuring charges in lung water, $J$. Microwave Power 18 (1983) 265.

[19] Enel, L., Leroy, Y., VAN de Velde, J. C., MAMOUNI, A., Improved recognition of thermal structures by microwave radiometry, Electron. Lett. 20 (1984) 293-294.

[20] Daburon, F., Lefaix, J. L., Remy, J., FAyart, G., Microwave radiometry of subcutaneous temperature evolution after acute local irradiation in pigs, Proc. 7th Int. Cong. Radiation Res. (Amsterdam) juillet 1983.

[21] Daburon, F., Lefaix, J. L., Remy, J., Fayart, G., Tricaud, Y., Mesures thermographiques microondes après irradiation localisée chez le porc: méthodes d'acquisition et de traitement, I.T.B.M. 8 (1987) 367-383.

[22] EDENHOFER, E., Electromagnetic remote sensing of the temperature profile by stochastic inversion, Radio Sci. 16 (1981) 1065-1069.

[23] SCHALlER, G., Inversion of radiometric data from biological tissue by an optimisation method, Electron. Lett. 20 (1984) 380-382.

[24] Chive, M., Plancot, M., Giaux, G., Prevost, B., Thermal dosimetry in microwave hyperthermia, 4th Int. Symp. Hyperthermie Oncology (Aarhus) 1984 , p. $863-866$.

[25]'Bardatti, F., Montgiardo, M., Solimini, D., Inversion of microwave thermography data by the singular function method. IEEE MTT's Digest (1985), p. 75-77.

[26] Bardatti, F., Calamini, G., Montgiardo, M., SoliminI, D., Multispectral radiometric system, Proc. 17th E.M.C. (Rome) 1987.

[27] Bocouet, B., MAMOUNI, M., Hochedez, M., VAN DE VELDE, J. C., LEROY, Y., Visibility of local thermal structures and temperature retrieval by microwave radiometry, Electron. Lett. 22 (1986) 120-122. 